\title{
Sucessão crambe-soja no manejo de Pratylenchus brachyurus e Meloidogyne javanica
}

\author{
Carolina Amaral Tavares-Silva ${ }^{1}$; Claudia Regina Dias-Arieira ${ }^{2}$; Heriksen Higashi Puerari²; Elizeu Junior da Silva ${ }^{2}$; \\ Adão Izidoro Junior ${ }^{1}$
}

${ }^{1}$ Universidade Paranaense, Departamento de Engenharia Agronômica, Umuarama, PR, Brasil; ${ }^{2}$ Universidade Estadual de Maringá, Pós Graduação em Agronomia, Maringá, PR, Brasil.

Autor para correspondência: Carolina Amaral Tavares da Silva (carolinaamaral@prof.unipar.br)

Data de chegada: 28/07/2016. Aceito para publicação em: 15/02/2017.

$10.1590 / 0100-5405 / 167249$

\section{RESUMO}

Tavares-Silva, C.A.; Dias-Arieira, C.R.; Puerari, H.H.; Silva E.J.; Izidoro Junior, A. Sucessão crambe-soja no manejo de Pratylenchus brachyurus e Meloidogyne javanica. Summa Phytopathologica, v.43, n.4, p.316-320, 2017.

A implantação do sistema de sucessão de culturas, possibilitando a inserção de espécies vegetais antagonistas ou não hospedeiras à patógenos da soja no período de entressafra, é uma alternativa sustentável em sistemas de cultivo. Neste contexto, recomenda-se cuidado na seleção de plantas de sucessão que não possibilitem a multiplicação de nematoides, especialmente, os parasitas da soja, Pratylenchus brachyurus e Meloidogyne javanica. Desta forma, objetivou-se avaliar o potencial de redução da população de $P$. brachyurus e $M$. javanica, pelo cultivo de espécies utilizadas em sucessão com a soja, comparando-as com o crambe. Sementes de soja cv. 5909RR Nidera foram semeadas em vasos com capacidade para $18 \mathrm{~L}$ e após 15 dias foram inoculadas, em experimentos separados, com 1000 espécimes de P. brachyurus e 3000 ovos e eventuais juvenis de segundo estádio (J2) de M. javanica. Aos 60 dias da inoculação, a parte aérea das plantas foi descartada e as culturas de sucessão (milho cv. IPR114, feijão cv. IPR Tangará, nabo forrageiro, aveia-preta cv. IAPAR 61 e crambe cv. MS Brilhante) foram semeadas. As plantas foram cultivadas por 90 dias, quando a parte aérea foi cortada, semeando-se novamente a soja, a qual foi cultivada por mais 60 dias. No final desse período, as plantas foram retiradas dos vasos para determinação de parâmetros vegetativos e nematológicos na soja. A introdução do crambe no sistema de sucessão de culturas reduziu a população de P. brachyurus. A reprodução de $M$. javanica foi menor na sucessão com o crambe quando comparado com os demais sistemas, entretanto o mesmo apresentou elevado fator de reprodução.

Palavras-chave: Crambe abyssinica, controle, nematoide das lesões radiculares, nematoide das galhas.

\section{ABSTRACT}

Tavares-Silva, C.A.; Dias-Arieira, C.R.; Puerari, H.H.; Silva E.J.; Izidoro Junior, A. Crambe-soybean succession on the management of Pratylenchus brachyurus and Meloidogyne javanica. Summa Phytopathologica, v.43, n.4, p.316-320, 2017.

Crop succession system implementation, which allows the introduction of plant species that are antagonists or non-hosts for soybean pathogens during the intercrop period, is a sustainable alternative in crop systems. Thus, careful selection of succession plants is recommended to ensure that they do not allow the proliferation of nematodes, especially the soybean parasites Pratylenchus brachyurus and Meloidogyne javanica. The aim of this study was to assess $P$. brachyurus and M. javanica population reduction potential by growing species in succession with soybean, comparing them to crambe. Soybean seeds cv. 5909RR Nidera were sown in 18-L pots, and after fifteen days inoculations were performed in separate experiments, with 1000 specimens of P. brachyurus and
3000 eggs and possible second-stage juveniles (J2) of M. javanica. At 60 days after inoculation, the shoot of soybean plants was discarded, and the succession crops (maize cv. IPR114, bean cv. IPR Tangará, oilseed radish, black oat cv. IAPAR 61 and crambe cv. MS Brilhante) were sown. Plants were cultivated for 90 days, when the shoot was removed, and soybean cultivar was sown again and cultivated for additional 60 days. At the end of this period, plants were removed from the pots for determination of vegetative and nematological parameters for soybean. Crambe introduction in the crop succession system effectively reduced $P$. brachyurus population. M. javanica reproduction was lower for crambe compared to the remaining systems, but its reproductive factor was high.

Keywords: Crambe abyssinica, control, root lesion nematode, root-knot nematode.

O cultivo da soja (Glycine max (L.) Merril) caracteriza-se como monocultura (23), que consiste no plantio da mesma espécie vegetal, no mesmo local da lavoura, onde estão presentes seus próprios restos culturais (34). De um lado o sistema apresenta vantagens, como avanços tecnológicos e outros benefícios; porém, pode gerar problemas fitossanitários, com aumento da infestação de agentes patogênicos e outras pragas (23).

Visando minimizar impactos fitossanitários negativos que a monocultura pode gerar, busca-se alternativas, como a sucessão de culturas, que é caracterizado como uma sequência pré-estabelecida de espécies vegetais, dentro do mesmo ano agrícola (35), possibilitando a inclusão de espécies antagonistas ou não hospedeiras de determinados fitopatógenos na entressafra da soja. Neste contexto, o cultivo de plantas de cobertura de inverno é uma opção importante, pois além de proteger o solo, contribui para a melhoria da qualidade estrutural do mesmo (40) e promove aumento da microbiota do solo e, com isso, a competição entre os microrganismos (5).

No sistema de cultivo brasileiro de soja, são comuns as sucessões de culturas, sendo as principais espécies vegetais utilizadas o milho (Zea mays L.) ou o trigo (Triticum aestivum L.) (18). Estas são bem estabelecidas, contudo, deve-se buscar alternativas, uma vez que tais plantas, especialmente o milho, pode multiplicar algumas espécies 
de nematoides importantes para a soja $(20,21)$, como o nematoide das lesões radiculares, Pratylenchus brachyurus (Godfrey) Filipjev e Schuurmans Steckhoven, considerada a espécie mais importante nas regiões produtoras de soja do país, e o nematoide das galhas, cuja espécie Meloidogyne javanica (Treub) Chitwood destaca-se entre as de grande importância para a soja $(16,20)$.

Apesar dos inúmeros trabalhos que mostram o potencial da sucessão para o controle destes nematoides na cultura da soja $(12,21,27,38$, 43), algumas espécies com potencial para sucessão como cultura de inverno ainda não foram pesquisadas, como é o caso do crambe (Crambe abyssinica Hoechst), uma Brassicaceae oleaginosa, cultivada no inverno, altamente resistente à seca e com ciclo curto (32), cujos grãos possuem alto teor de óleo, que serve como fonte de matéria prima para a produção de biodiesel (31). A hipótese que esta planta apresenta potencial para o manejo de nematoides, baseia-se nos fatos de outras brássicas, como o nabo forrageiro, apresentarem eficiência para este fim $(12,38,43)$ e que as plantas desta família botânica sintetizam compostos nematicidas $(28,37,45)$.

Assim, o trabalho teve como objetivo avaliar, em condições controladas, a sucessão de culturas de soja com aveia-preta (Avena strigosa Schreb), nabo forrageiro (Raphanus sativus L. var. oleiferus Metzg), milho e feijão (Phaseolus vulgaris L.), comparando-as com o crambe, sobre as populações de $P$. brachyurus e $M$. javanica.

\section{MATERIAL E MÉTODOS}

Os experimentos foram conduzidos em casa de vegetação, na Universidade Estadual de Maringá, Campus Regional de Umuarama, Umuarama, Paraná, Brasil. A condução do experimento com $P$. brachyurus foi entre abril e dezembro de 2013 e o de M. javanica entre junho de 2013 e janeiro de 2014. Adotou-se delineamento inteiramente casualizado, com seis tratamentos e seis repetições.

Sementes de soja cv. 5909RR Nidera foram semeadas em vasos contendo $15 \mathrm{~L}$ de solo previamente autoclavado $\left(120^{\circ} \mathrm{C} / 2 \mathrm{~h}\right)$ e, posteriormente, raleadas mantendo uma planta por vaso. O solo utilizado como substrato foi classificado como Latossolo Vermelho distrófico típico, de textura arenosa, e foi adubado de acordo com análise de solo e necessidade da cultura da soja. Após 15 dias da semeadura, as plantas foram inoculadas, em experimentos separados, com 1000 indivíduos de $P$. brachyurus ou 3000 ovos e eventuais juvenis de segundo estádio (J2) de M. javanica. Os inóculos foram obtidos de populações puras mantidas em milho e tomateiro, respectivamente, e foram extraídos pelos métodos propostos Coolen \& D'herde (13) e Hussey \& Barker (19), respectivamente, sendo quantificados em câmara de Peters, sob microscópio óptico. As populações utilizadas foram diferenciadas em função da disponibilidade de inóculos no momento da extração

Aos 60 dias da inoculação, a parte aérea da soja foi descartada e as culturas de sucessão: aveia-preta cv. IAPAR 61, crambe cv. MS Brilhante, nabo forrageiro, milho cv. IPR114 e feijão cv. IPR Tangará foram semeadas. Vasos sem plantas contendo os nematoides foram mantidos em ambos os experimentos, simulando o solo em pousio. Seguindo recomendações de Calegari (8) e Pitol et al. (32), foram semeadas 20 sementes/vaso para aveia-preta e 15 sementes/vaso para crambe e nabo forrageiro. A densidade de semeadura do milho (17) e feijão (39) foi de duas plantas/vaso, mantendo-se a mesma quantidade de plantas semeadas.

As plantas foram cultivadas por 90 dias, quando tiveram a parte aérea cortada, picada e mantidas na superfície do solo. A mesma cultivar de soja foi semeada, mantendo-se uma planta por vaso após desbaste. Decorridos 60 dias, as plantas foram retiradas dos vasos, determinando-se a altura da parte aérea, massa fresca da parte aérea e da raiz e massa seca da parte aérea, sendo esta obtida por secagem em estufa de circulação forçada, a $65^{\circ} \mathrm{C}$ por três dias. As raízes foram submetidas às extrações de nematoides, conforme metodologias citadas, e avaliou-se o número de nematoide por sistema radicular. Posteriormente, este foi dividido pela massa da raiz, obtendo-se o número de nematoide/g de raiz.

Os dados foram submetidos à análise de variância e as médias comparadas pelo teste de Duncan a 5\% de erro. Para análise estatística, os parâmetros nematoides/g de raiz e número total de nematoides na planta (M. javanica), foram transformados pela $\sqrt{ }(\mathrm{x}+1)$.

\section{RESULTADOS E DISCUSSÃO}

No experimento com $P$. brachyurus, a sucessão de culturas não interferiu nos parâmetros vegetativos de parte aérea ou na massa de raiz da soja (Tabela 1). Quanto aos parâmetros nematológicos, as sucessões com milho e com aveia resultaram nos maiores valores de população total, mas a aveia não diferiu estatisticamente dos demais tratamentos (Tabela 2). O tratamento com milho apresentou média de nematoides/g de raiz $(289,9)$ superior ao crambe $(74,5)$.

Tabela 1. Altura de planta, massa da parte aérea fresca (MF) e seca (MS) e da raiz da soja aos 60 dias de cultivo, após sucessão de culturas, em solo infestado com P. brachyurus.

\begin{tabular}{lcccc}
\hline Tratamentos & Altura (cm) & MF (g) & MS (g) & MFR (g) \\
\hline Milho & $36,9^{\text {ns }}$ & $40,8^{\text {ns }}$ & $10,0^{\text {ns }}$ & $7,5^{\text {ns }}$ \\
Aveia-preta & 28,8 & 27,8 & 8,3 & 6,1 \\
Nabo forrageiro & 32,3 & 32,5 & 9,1 & 6,2 \\
Feijão & 33,8 & 30,5 & 8,6 & 4,4 \\
Pousio & 30,0 & 35,7 & 9,9 & 3,8 \\
Crambe & 30,7 & 32,5 & 9,5 & 4,4 \\
\hline CV(\%) & 30,9 & 42,9 & 39,3 & 36,0
\end{tabular}

Médias seguidas pela mesma letra nas colunas não diferem entre si pelo teste de Duncan a $5 \%$ de probabilidade. $n s=$ não significativo. $\mathrm{CV}=$ coeficiente de variação.

A sucessão com aveia-preta cv. Iapar 61 resultou em aumento da população final de $P$. brachyurus, porém se comparada ao milho, houve redução de 42,3 e $28,8 \%$ no número total de $P$. brachyurus e de nematoide/g de raiz, respectivamente (Tabela 2). Por outro lado, o nabo forrageiro foi eficiente no controle de P. brachyurus, reduzindo em 46,9 e $36,0 \%$ a população total e nematoide/g de raiz, respectivamente. Semelhantemente, a população de $P$. brachyurus diminuiu na sucessão soja-feijão-soja (56,8 e 26,2\%, para população total e nematoide/g de raiz) e na sucessão soja-crambe-soja, com respectivas reduções de 84,8 e 74,3\% (Tabela 2).

O aumento populacional de $P$. brachyurus na sucessão soja-milhosoja confirma os relatos de outros autores $(14,20,29,43)$, sendo que este mesmo sistema de cultivo promoveu aumento no número de nematoides/g de raiz, quando comparado à sucessão com crotalária (Crotalaria juncea L.), estilosantes (Stylosantes capitata Vog. + Stylosantes macrocephala Ferreira e Costa $(80+20 \%)$ ) e pousio (38). 
Tabela 2. População total, nematoide g de raiz e porcentagem de redução em relação ao milho (RRM) de P. brachyurus em sistema de sucessão de culturas soja-tratamento-soja.

\begin{tabular}{lcccc}
\hline Tratamentos & Pop. total & \%RRM & Nema/g raiz & \%RRM \\
\hline Milho & $2.177,5 \mathrm{a}$ & - & $289,9 \mathrm{a}$ & - \\
Aveia-preta & $1.256,2 \mathrm{ab}$ & 42,3 & $206,4 \mathrm{ab}$ & 28,8 \\
Nabo & $1.157,0 \mathrm{~b}$ & 46,9 & $185,6 \mathrm{ab}$ & 36,0 \\
Feijão & $940,0 \mathrm{~b}$ & 56,8 & $214,0 \mathrm{ab}$ & 26,2 \\
Pousio & $736,5 \mathrm{~b}$ & 66,2 & $194,8 \mathrm{ab}$ & 32,8 \\
Crambe & $330,0 \mathrm{~b}$ & 84,8 & $74,5 \mathrm{~b}$ & 74,3 \\
\hline CV(\%) & 50,8 & - & 54,1 & - \\
\hline
\end{tabular}

Médias seguidas pela mesma letra nas colunas não diferem entre si pelo teste de Duncan a $5 \%$ de probabilidade. $\mathrm{CV}=$ coeficiente de variação.

Este fato é relativamente comum e tal sucessão tem sido apontada como responsável pelo aumento de P. brachyurus em área de cultivo de soja (20).

Constatou-se que apesar da sucessão com aveia-preta cv. Iapar 61 ter promovido aumento da população final de $P$. brachyurus, possibilitou a redução do nematoide, quando comparada ao milho. Anteriormente, esta cultivar promoveu redução do nematoide em experimento de campo e casa de vegetação (12), sendo a aveia preta considerada uma hospedeira desfavorável a esta espécie de nematoide (6).

Diferentemente, o nabo forrageiro reduziu a população de $P$. brachyurus, confirmando dados apresentados por outros autores, os quais indicaram esta espécie vegetal como resistente ao nematoide, com fatores de reprodução (FR) variando de 0,03 a $0,35(12,22)$. Contudo, em trabalhos anteriores, apesar do nabo forrageiro reduzir a população de $P$. brachyurus, ele foi menos eficiente que outras espécies como mucuna (Stizolobium aterrimum Piper and Tracy) cv. Mucunapreta, crotalária, estilosantes e guandu (Cajanus cajan (L.) Mill) cv. Caqui (43). A população total de $P$. brachyurus também diminuiu na sucessão soja-feijão-soja, resultados que discordam dos trabalhos de outros autores, que observaram que o feijão foi suscetível ao nematoide, aumentando sua população final (1). Dois fatores podem ter contribuído para estas diferenças, a densidade populacional do nematoide no solo (24) e a cultivar utilizada (1). Amaro et al. (1) observaram que a cv. Tangará, a mesma utilizada neste trabalho, foi suscetível a $P$. brachyurus $(\mathrm{FR}=2,71)$, contudo apresentou FR inferior a maioria das cultivares avaliadas.

O pousio do solo após a colheita da soja promoveu a redução na população de nematoide das lesões radiculares, confirmando os relatos de Barry \& Rhodes (4) e Rodrigues et al. (38), que observaram o decréscimo da população de P. bachyurus em sistemas de solo em pousio. Este é uma opção de manejo de nematoides, porém, é uma prática de difícil aplicabilidade, em razão do uso constante da mesma área e pela necessidade de manter controle rigoroso de plantas daninhas suscetíveis ao nematoide (10). Além disto, pode acarretar problemas de erosão, diminuição da fertilidade do solo, pelo decréscimo de matéria orgânica, e redução de nutrientes e microrganismos benéficos (26).

A sucessão soja-crambe-soja apresentou os melhores resultados para o controle de P. brachyurus, dados que estão de acordo com Dias et al. (15), os quais observaram que o crambe foi imune ao fitoparasito, com FR igual a zero. Possivelmente, o efeito do crambe, a exemplo do que ocorre com outras brássicas, seja a combinação entre a capacidade dessas espécies atuarem como plantas-armadilhas e a liberação de compostos nematotóxicos, principalmente o isotiocianato, via exsudatos radiculares ou durante a decomposição dos resíduos vegetais (7).

No experimento com $M$. javanica, observou-se que a sucessão de culturas não influenciou estatisticamente os parâmetros vegetativos, como altura de planta e massa fresca e seca da parte aérea da soja (Tabela 3). A massa de raiz das plantas da soja diferiu entre os tipos de sucessão de culturas (Tabela 3), sendo que a sucessão soja-crambesoja influenciou positivamente $(64,3 \mathrm{~g})$ e a soja-aveia preta-soja negativamente $(29,5 \mathrm{~g})$, porém, ambas não diferiram das demais culturas.

Tabela 3. Médias de altura de planta, massa da parte aérea fresca (MF) e seca (MS) da soja em função das plantas em sucessão em vasos inoculados com M. javanica.

\begin{tabular}{lcccc}
\hline Tratamentos & Altura $(\mathbf{c m})$ & MF $(\mathbf{g})$ & MS $(\mathbf{g})$ & MFR $(\mathbf{g})$ \\
\hline Milho & $51,3^{\text {ns }}$ & $35,2^{\text {ns }}$ & $9,6^{\text {ns }}$ & $34,7 \mathrm{ab}$ \\
Aveia-preta & 49,4 & 19,5 & 5,0 & $29,5 \mathrm{~b}$ \\
Nabo forrageiro & 55,6 & 32,7 & 8,7 & $48,9 \mathrm{ab}$ \\
Feijão & 52,5 & 36,4 & 9,8 & $52,3 \mathrm{ab}$ \\
Pousio & 54,0 & 33,4 & 9,0 & $44,9 \mathrm{ab}$ \\
Crambe & 56,5 & 39,6 & 10,5 & $64,3 \mathrm{a}$ \\
\hline CV(\%) & 18,6 & 34,9 & 28,3 & 26,0 \\
\hline
\end{tabular}

Médias seguidas pela mesma letra nas colunas não diferem entre si pelo teste de Duncan a 5\% de probabilidade. ns= não significativo. $\mathrm{CV}=$ coeficiente de variação.

A população total de $M$. javanica aumentou em todos os sistemas de sucessão quando comparado com a população inicial, que foi de 3000 ovos e J2 no momento da inoculação, sendo o feijão a cultura que possibilitou maior aumento na população total de $P$. brachyurus (Tabela 4). O milho foi o tratamento que resultou em menor aumento e promoveu redução de $58,5 \%$ na população total, se comparado ao feijão, cultura que possibilitou maior reprodução do nematoide.

Tabela 4. População total, nematoide g de raiz e porcentagem de redução em relação ao milho (RRM) de $M$. javanica em sistema de sucessão de culturas soja-tratamento-soja.

\begin{tabular}{lcccc}
\hline Tratamentos & Pop. total & \%RRF & Nema/g raiz & \%RRF \\
\hline Milho & $194.560 \mathrm{~b}$ & 58,5 & $5.417 \mathrm{bc}$ & 44,2 \\
Aveia-preta & $334.847 \mathrm{ab}$ & 28,6 & $12.370 \mathrm{a}$ & $-27,3$ \\
Nabo forrageiro & $269.192 \mathrm{ab}$ & 42,6 & $6.881 \mathrm{abc}$ & 29,1 \\
Feijão & $469.125 \mathrm{a}$ & - & $9.713 \mathrm{ab}$ & - \\
Pousio & $324.667 \mathrm{ab}$ & 30,8 & $8.037 \mathrm{abc}$ & 17,2 \\
Crambe & $271.340 \mathrm{ab}$ & 42,2 & $4.505 \mathrm{c}$ & 53,6 \\
\hline CV(\%) & 32,0 & - & 36,0 & -
\end{tabular}

Médias seguidas pela mesma letra nas colunas não diferem entre si pelo teste de Duncan a 5\% de probabilidade. CV = coeficiente de variação.

As demais culturas não diferiram do milho e do feijão quanto ao número total de nematoides. Porém, o crambe apresentou o menor número de nematoides/g de raiz se comparado a outras plantas estudadas, especialmente o feijão, cuja reduções foram de 42,2 e 53,6\% no número total de ovos e de ovos/g de raiz (Tabela 4).

No experimento com $M$. javanica, o milho promoveu redução na população total de nematoide, se comparado ao feijão, tratamento que permitiu maior aumento do nematoide. De fato, algumas cultivares de 
milho podem atuar como hospedeiro desfavoráveis aos nematoides das galhas (3), sendo observada a resistência da maioria dos 85 genótipos de milho a $M$. javanica, cujos FRs variaram de 0,02 a 5,68, enquanto as testemunhas apresentaram FRs de 43,28 a 104,53 (36).

Da mesma forma, o aumento da população de $M$. javanica na sucessão soja-feijão-soja, confirma as pesquisas que classificaram várias cultivares desta espécie como suscetível ao nematoide das galhas $(41,46)$. Os demais tratamentos, como aveia preta, nabo forrageiro, pousio e crambe, não diferiram significativamente das sucessões com milho e feijão, dados que discordam de relatos anteriores, em que algumas destas plantas foram consideradas como não hospedeiras $(9$, 11). Estes relatos controversos apontam para a necessidade de cautela ao utilizar determinadas espécies vegetais em esquemas de rotação de culturas visando o manejo de nematoides das galhas.

O crambe não reduziu a população final do nematoide, porém apresentou o menor número de nematoides/g de raiz se comparado aos demais tratamentos. É possível que tais reduções tenham proporcionado maior desenvolvimento das raízes da soja. Além disto, a decomposição de algumas brássicas libera compostos tóxicos com comprovado efeito nematicida, como os glucosilonatos, presentes nas raízes e tecidos vegetais, os quais são hidrolisados pela enzima mironase, produzindo diferentes isoticionatos, quimicamente similares ao isoticionato de metila, fumigante de solo de amplo espectro $(25,30,33,44)$. Mas, vale ressaltar a necessidade de cuidado com o uso desta espécie em áreas infestadas por Meloidogyne spp., visto que a planta foi considerada suscetível a estes parasitas $(2,42)$.

A sucessão soja-crambe-soja foi eficiente na redução da população de $P$. brachyurus, em aproximadamente $85 \%$ quando comparado com a sucessão soja-milho-soja. O crambe reduziu em mais de 50,0\% no número de ovos de $M$. javanica/g de raiz de na sucessão com a soja, se comparado a sistema de sucessão soja e feijão.

\section{REFERÊNCIAS}

1. Amaro, P.M.; Matunaga, D.S.; Marini, P.M.; Silva, S.A.; Machado, A.C.Z. Reação de cultivares de feijoeiro a Meloidogyne incognita e Pratylenchus brachyurus. In: Congresso Nacional de Pesquisa de Feijão, 11., 2014, Londrina. Anais. Londrina: Iapar, 2014. p.1-4.

2. Asmus, G.L.; Andrade, P.J.M. Reprodução do nematoide das galhas (Meloidogyne javanica) em algumas plantas alternativas para uso em sucessão à cultura da soja. Comunicado Técnico, Embrapa Agropecuária Oeste, Dourados, v.37, p.1-4, 2001

3. Asmus, G.L.; Ferraz, L.C.C.B.; Appezzato-da-Glória, B. Alterações anatômicas em raízes de milho (Zea mays L.) parasitadas por Meloidogyne javanica. Nematropica, Auburn, v.30, n.1, p.33-40, 2000.

4. Barry, S.; Rhodes, R. Green manure crops: agronomic characteristics and effect on nematodes. South African Sugar Technologists' Association, Mount Edgecomb, v.80, p.269-273, 2006.

5. Bettiol, W.; Ghini, R. Solos supressivos. In: Michereff, S.J.; Andrade D.E.G.T.; Menezes, M. (Ed.). Ecologia e manejo de patógenos radiculares em solos tropicais. Recife: UFRPE, 2005. p.125-152.

6. Borges, D.C.; Machado, A.C.Z.; Inomoto, M.M. Reação de aveias a Pratylenchus brachyurus. Tropical Plant Pathology, Brasília, DF, v.35, n.3, p.178-181, 2010.

7. Brown, P.D.; Morra, M.J. Control of soil-borne plant pests using glucosinolate-containing plants. In: Sparks, D.L. (Ed.). Advances in Agronomy. San Diego: Academic Press, 1997. p.167-215.

8. Calegari, A. Plantas de cobertura. In: Casão Junior, R.; Siqueira, R.; Mehta, Y. R.; Passini, J.J. Plantio direto com qualidade. Londrina: IAPAR; Foz do Iguaçu: ITAIPU Binacional, 2006. p.55-73,

9. Carneiro, R.M.D.G.; Carvalho, F.L.C.; Kulczynski, S.M. Seleção de plantas para o controle de Mesocriconema xenoplax e Meloidogyne spp. através de rotação de culturas. Nematologia Brasileira, Piracicaba, v.22, n.2, p.41-48, 1998.

10. Castro, A.P.; Santos, M.A. Reprodução de Pratylenchus brachyurus em plantas de soja sob condições de casa de vegetação a partir de diferentes populações iniciais de inóculo. Horizonte Científico, Uberlândia, n.8, v.1, 2008. Disponível em: $<$ http://www.horizontecientifico.propp.ufu.br/include/getdoc.php?id=710\&article=288mode=pdf $>$. Acesso em: 03 ago. 2014 .

11. Charchar, J.M.; Gonzaga, V.; Vieira, J.V.; Oliveira, V.R.; Moita, A.W.; Aragão, F. A. S. Efeito da rotação de culturas no controle de Meloidogyne spp. em cenoura na região norte do estado de Minas Gerais. Nematologia Brasileira, Piracicaba, v.31, n.3, p.173-179, 2007.

12. Chiamolera, F.M.; Dias-Arieira, C.R.; Souto, E.R.; Biela, F.; Cunha, T.P.L.; Santana, S. M.; Puerari, E. H. Suscetibilidade de culturas de inverno a Pratylenchus brachyurus e atividade sobre a população do nematoide na cultura do milho. Nematropica, Auburn, v.42, n.2, p.267-275, 2012.

13. Coolen, W.A.; D'Herde, C.J.A. Method for the quantitative extraction of nematodes from plant tissue. Ghent: State Nematology and Entomology Research Station. 1972. 77p.

14. Costa, M.J.N.; Pasqualli, R.M.; Prevedello, R. Efeito do teor de matéria orgânica do solo, cultura de cobertura e sistema de plantio no controle de Pratylenchus brachyurus em soja. Summa Phytopathologica, Botucatu, v.40, n.1, p.63-70, 2014.

15. Dias, W.P.; Orsini, I.P.; Ribeiro, N.R.; Parpinelli, N.M.B.; Freire, L. L. Efeito do cultivo de espécies vegetais sobre a população de Pratylenchus brachyurus na soja. In: Congresso Brasileiro de Soja, 6., 2012, Cuiabá Anais. Cuiabá: Embrapa, 2012. p. 1-4.

16. Dias, W.P.; Asmus, G.L.; Silva, J.F.V.; Garcia, A.; Carneiro, G.E.S. Nematoides. In: Almeida, A.M.R.; Seixas, C.D.S. (Ed.). Soja: doenças radiculares e de hastes e interrelações com o manejo do solo e da cultura. Embrapa Soja: Londrina, 2010. p.173-206.

17. Fancelli, A.L.; Dourado Neto, D. Produção de milho. Guaiba: Agropecuária, 2000. 360p.

18. Franchini, J.C.; Costa, J.M.; Debiasi, H. Rotação de culturas: Prática que confere maior sustentabilidade à produção agrícola no Paraná. Informações Agronômicas, Piracicaba, v.134, p.1-13, 2011.

19. Hussey, R.S.; Barker, K.R.A comparison of methods of collecting inocula of Meloidogyne spp., including a new technique. Plant Disease Reporter, Beltsville, v.57, p.1025-1028, 1973.

20. Inomoto, M.M. Avaliação da resistência de 12 híbridos de milho a Pratylenchus brachyurus. Tropical Plant Pathology, Brasília, DF, v.36, p.308-312, 2011.

21. Inomoto, M.M.; Antedomênico, S. R.; Santos, V. P.; Silva, R. A.; Almeida, G. C. Avaliação em casa de vegetação do uso de sorgo, milheto e crotalária no manejo de Meloidogyne javanica. Tropical Plant Pathology, Brasília, DF, v.33, n.2, p.125-129, 2008.

22. Inomoto, M.M.; Motta, L.C.C.; Beluti, D.B.; Machado, A.C.Z. Reação de seis adubos verdes a Meloidogyne javanica e Pratylenchus brachyurus. Nematologia Brasileira, Piracicaba, v.30, n.1, p.39-44, 2006.

23. Kuhn, R.A.; Portz, R.L.; Stangarlin, J.R. Uso de biomassa cítrica no controle de doenças da soja. Scientia Agraria Paranaensis, Marechal Cândido Rondon, v.8, p.85-89, 2009.

24. Machado, A.C.Z.; Marini, P.M.; Dadazio, T.S.; Silva, S.A. Patogenicidade de Pratylenchus brachyurus em feijão. In: Congresso Nacional de Pesquisa de Feijão, 11., 2014, Londrina. Anais. Londrina: Iapar, 2014. p.1-4.

25. McCully, M.E.; Miller, C.; Sprague, S.J.; Huang, C.X.; Kirkegaard, J.A. Distribution of glucosinolates and sulphur-rich cells in roots of field-grown canola (Brassica napus). New Phytologist, Boston, v.180, p.193-205, 2008.

26. McSorley, R.; Gallaher, R.N. Effect of tillage and crop residue management on nematode densities on corn. Journal of Nematology, St. Paul, v.9, p.731-736, 1994.

27. Mendes, F.L.; Araújo, K.; Debiasi, H.; Franchini, J.C.; Dias, W.P.; Ramos Junior, E.U.; Silva, J.F.V. Alternativas culturais para o manejo do nematoide das lesões radiculares durante a entressafra da soja no Mato Grosso. In: Jornada Acadêmica da Embrapa Soja, 8., 2013, Londrina. Resumos expandidos. Londrina: Embrapa Soja, 2013. p.97-103.

28. Neves, W.S.; Freitas, L.G.; Coutinho, M.M.; Parreira, D.F.; Ferraz, S.; Costa, M. D. Biofumigação do solo com espécies de brássicas para o controle de Meloidogyne javanica. Nematologia Brasileira, Piracicaba, v.31, n.3, p.195-201, 2007.

29. Nikuma, H.H.; Dias, I.; Paes, V.S.; Silva, A.; Vendramini, A.; Soares, P.L.M. Avaliação da resistência de genótipos de milho e sorgo ao nematoide das lesões, Pratylenchus brachyurus. Ciência e Tecnologia, Jaboticabal, v.4, p.1-5, 2012. Suplemento.

30. Oka, Y. Mechanisms of nematode suppression by organics oil amendments-A 
review. Applied Soil Ecology, Firenze, v.44, p.101-115, 2010.

31. Oplinger, E.S.; Oelke, E.A.; Kaminski, A.R.; Putman, D.H.; Teynor, T.M.; Doll, J.D.; Kelling, K.A.; Durgan, B.R.;Noetzel, D.M. Crambe, alternative field crops manual. St. Paul: University of Wisconsin and University of Minnesota, 1991.

32. Pitol, C.; Broch, D.L.; Roscoe, R. Tecnologia e produção: crambe. Maracajú: Fundação MS, 2010. 60p.

33. Ratnadass, A. Fernandes, P.; Avelino, J.; Habib, R. Plant species diversity for sustainable management of crop pests and diseases in agroecosystems: a review. Agronomy for Sustainable Development, Paris, v.32, p.273303, 2012.

34. Reis, E.M.; Casa, R.T.; Bianchin, V. Controle de doenças de plantas pela rotação de culturas. Summa Phytopathologica, Botucatu, v.37, n.3, p.8591, 2011.

35. Reis, E.M.; Casa, R.T.; Hoffmann, L.L. Controle cultural de doenças radiculares. In: Michereff, S.J.; Andrade, D.E.G.T., Menezes, M. (Ed.). Ecologia e manejo de patógenos radiculares em solos tropicais. Recife: UFRPE, 2005. p. 279-301.

36. Ribeiro, N.R.; Craveiro, A.G.; Silva, J.F.V.; Francisco, A.; Gomes, J.; Meirelles, W.F. Avaliação de genótipos de milho (Zea mays) aos nematoides Meloidogyne javanica e M. incognita Raça 3. In: Congresso Nacional de Milho e Sorgo, 24., 2002, Florianópolis. Anais. Florianópolis: Embrapa, 2002. p.1-5.

37. Rizzardi, M.A.; Neves, R.; Lamb, T.D.; Johann, L.B. Potencial alelopático da cultura da canola (Brassica napus L. var. oleífera) na supressão de picão-preto (Bidens sp.) e soja. Revista Brasileira. Agrociência, Pelotas, v.14, n.2, p.239-248, 2008.

38. Rodrigues, D.B., Dias-Arieira, C.R.; Vedoveto, M.V.V.; Roldi, M.; Molin, H.F. D.; Abe, V.H.F. Sucessão de culturas no manejo de Pratylenchus bra- chyurus em soja. Nematropica, Auburn, v.43, n.1, p.79-93, 2014.

39. SILVA, C. C. da. Plantio. In: AIDAR, H. (Ed.). Cultivo do feijoeiro comum. Santo Antônio de Goiás: Embrapa Arroz e Feijão; Campinas: Embrapa Informática Agropecuária, 2003. (Embrapa Arroz e Feijão. Sistemas de produção, 2)

40. Silva, V.L.; Dieckow, J.M.; Molin, R.; Favaretto, N.; Pauletti, V.; Vezzani, F. M. Melhoria da estrutura de um Latossolo por sistemas de culturas em plantio direto nos Campos Gerais do Paraná. Revista Brasileira de Ciência do Solo, Viçosa, v.36, n.3, p.983-992, 2012.

41. Simão, G.; Homechin, M.; Santiago, D.C.; Silva, R.T.V.; Ribeiro, E.R. Comportamento de duas cultivares de feijoeiro em relação à Meloidogyne javanica. Ciência Rural, Santa Maria, v.35, n.2, p.266-270, 2005.

42. Souza, R.A.; Ribeiro, R.C.F.; Rocha, L.S.; Xavier, A.A.; Soares-Martins, I.P.; Silva, F. J. Reação de crambe (Crambe abyssinica Hochst) à Meloidogyne javanica. In: Congresso Brasileiro de Fitopatologia, 44., 2011, Bento Gonçalves. Anais. Bento Gonçalves: Sociedade Brasileira de Fitopatologia, 2011. p.781.

43. Vedoveto, M.V.V.; Dias-Arieira, C.R.; Rodrigues, D.B.; Arieira, J.O.; Roldi, M.; Severino, J.J. Adubos verdes no manejo de Pratylenchus brachyurus em soja. Nematropica, Auburn, v.43, n.2, p.226-232, 2013.

44. Wu, H.; Wang, C.; Bian, X.; Zeng, S.; Lin, K.; Wu, B.; Zhang, G.; Zhang, $\mathrm{X}$. Nematicidal efficacy of isothiocyanates against root-knot nematode Meloidogyne javanica in cucumber. Crop Protection, Queensland, v.30, n.1, p.33-37, 2011.

45. Zasada, I.A.; Ferris, H. Nematode suppression with brassicaceous amendments: application based upon glucosinolate profiles. Soil Biology e Biochemistry, Leicestershire, v.36, p.1017-1024, 2004.

46. Zeni, F.; Dadazio, T.S.; Amaro, P.M.; Silva, S.A.; Machado, A.C.Z. Reação de cultivares de feijão a Meloidogyne javanica. In: Congresso Nacional de Pesquisa de Feijão, 11., 2014, Londrina. Anais. Londrina: Iapar, 2014. p.1-4. 